\title{
Perihematomal edema as predictor of outcome
}

Intracerebral hemorrhage $(\mathrm{ICH})$ is the most serious form of stroke, with over two-thirds of patients either dying or left permanently disabled from the condition. It is well recognized that the location, initial volume at presentation, and subsequent degree of growth of the hematoma of $\mathrm{ICH}$ are critical determinants of the prognosis, for which early intensive blood pressure (BP) lowering $^{[1]}$ and targeted surgical decompression ${ }^{[2]}$ offer the best opportunities to benefit the patient. As perihematomal edema, a component of $\mathrm{ICH}$, contributes to the resulting mortality and morbidity, it lends itself to being an additional therapeutic target in this disease. However, it is a complex process that involves several pathophysiological mechanisms, ranging from the hydrostatic pressure of the hematoma to subsequent toxic effects of breakdown products resulting from coagulation cascade activation and erythrocyte lysis as part of the natural process of hematoma resolution. ${ }^{[3]}$ Thus, although perihematoma edema and hematoma volumes are strongly correlated, there is less conclusive evidence regarding role of perihematomal edema itself as being an independent prognostic factor over and above the hematoma.

The article by Gupta et al. provides further support for the hematoma being the pivotal prognostic determining factor in $\mathrm{ICH}$, but it also suggests that relative perihematomal edema is a good prognostic sign. ${ }^{[4]}$ These latter findings are somewhat counter-intuitive, as they imply that patients with greater cerebral edema response to the underlying hematoma have a better outcome. However, it could also imply that patients with early cessation of hemorrhage, or clot retraction, relative to the surrounding edema, have better outcomes. There is some support for this hypothesis in a study showing that baseline relative perihematomal edema ( $<20$ hours of onset) was strongly associated with improved functional outcome (odds ratio [OR] 0.79 per

\begin{tabular}{|l|l|}
\hline \multicolumn{2}{|c|}{ Access this article online } \\
\hline Quick Response Code: & Website: \\
\hline & www.ruralneuropractice.com \\
\cline { 2 - 3 } & \\
\hline & \\
\hline
\end{tabular}

$10 \%$ increase; $P=0.02$ ) but not mortality. ${ }^{[5]}$ However, another study indicates that relative perihematomal edema at presentation predicts early neurological deterioration (OR 22.6, $P=0.009){ }^{[6]}$ while other studies found that relative edema at 48-72 hours had no relation with either early neurologic deterioration ${ }^{[7]}$ or death/ dependency at 90 days. ${ }^{[8]}$

Methodological differences in definitions, case selection, timing and measures of outcome likely contribute to the discrepancies in results across studies. It is quite plausible, though, that the etiology and significance of, and thus therapeutic approach toward, perihematomal edema will differ over the course of $\mathrm{ICH}$. Early after the onset of $\mathrm{ICH}$, the degree of perihematomal edema follows closely with the volume of hematoma, as it is directly related to extraversation of serum from the pressure of the hematoma. In which case, reduction (even decompression) of hematoma is the most direct therapeutic approach. Beyond 72 hours, though, perihematomal edema has a strong component of toxicity from the iron, thrombin, and other blood products, as well as local natural reactive inflammation. While subsidiary analysis of the INTERACT2 trial will likely shed light on any benefits of effective control of BP beyond the first 24 hours, other therapeutic approaches at this time that are under investigation include the use of iron chelation with deferroxamine. ${ }^{[9]}$

Current treatment of perihematoma edema is directed at managing raised intracranial pressure (ICP), and especially if there is hydrocephalus. The principles of managing ICP in ICH are borrowed from experience in traumatic brain injury where there is a strong emphasis on maintaining adequate cerebral perfusion pressure according to the status of cerebral autoregulation, although there is much controversy over the use of ICP monitoring at all, let alone by what is the most appropriate location site in the brain. ${ }^{[10]} \mathrm{ICH}$ patients with a Glasgow Coma Scale score of 8 or less, evidence of transtentorial herniation, significant intraventricular hemorrhage or hydrocephalus, should be considered for ICP monitoring and aggressive treatment. Further research is required to better elucidate the prognostic significance of perihematoma edema in ICH and to translate the mechanistic data into the clinical domain. 


\section{Craig Anderson}

The George Institute for Global Health, University of Sydney and Royal Prince Alfred Hospital, Sydney, Australia

Address for correspondence: Dr. Craig Anderson,

The George Institute for Global Health, Australia, Level 10, King George V Building, 83-117 Missenden Rd Camperdown NSW 2050 Australia, PO Box M201, Missenden Rd NSW 2050 Australia. E-mail: canderson@georgeinstitute.org.au

\section{References}

1. Anderson CS, Heeley E, Huang Y, Wang J, Stapf C, Delcourt C, et al. Rapid blood pressure lowering in acute intracerebral hemorrhage. N Engl J Med 2013;368:2355-65.

2. Gregson BA, Broderick JP, Auer LM, Batjer H, Chen XC, Juvela S, et al. Individual patients data subgroup meta-analysis of surgery for spontaneous supratentorial intracerebral hemorrhage. Stroke 2012;43:1496-504.

3. Wei JW, Arima H, Anderson CS. Significance of perihematomal edema in acute intracerebral hemorrhage. Euro Neurol J 2010;2:120-31.

4. Gupta M, Verma R, Parihar A, Garg RK, Singh MK. Perihematomal edema as predictor of outcome in spontaneous intracerebral hemorrhage J Neurosci Rural Pract 2014;5:48-55.

5. Gebel JM Jr, Jauch EC, Brott TG, Khoury J, Sauerbeck L, Salisbury S, et al. Relative edema volume is a predictor of outcome in patients with hyperacute spontaneous intracerebral hemorrhage. Stroke 2002;33:2636-41.

6. Sykora M, Diedler J, Turcani P, Rupp A, Steiner T. Subacute perihematomal edema in intracerebral hemorrhage is associated with impaired blood pressure regulation. J Neurol Sci 2009;284:108-12.

7. Leira R, Davalos A, Silva Y, Gil-Peralta A, Tejada J, Garcia M, et al. Early neurologic deterioration in intracerebral hemorrhage: Predictors and associated factors. Neurology 2004;63:461-7.

8. Arima H, Wang JG, Huang Y, Heeley E, Skulina C, Parsons MW, et al. Significance of perihematomal edema in acute intracerebral hemorrhage: The INTERACT trial. Neurology. 2009;73:1963-8.

9. Selim M, Yeatts S, Goldstein JN, Gomes J, Greenberg S, Morgenstern LB, et al. Safety and tolerability of deferoxamine mesylate in patients with acute intracerebral hemorrhage. Stroke 2011;42:3067-74.

10. Chesnut RM, Temkin N, Carney N, Dikmen S, Rondina C, Videtta W, et al. A trial of intracranial-pressure monitoring in traumatic brain injury. N Engl J Med 2012;367:2471-81.

How to cite this article: Anderson C. Perihematomal edema as predictor of outcome. J Neurosci Rural Pract 2014;5:1-2. Source of Support: Nil. Conflict of Interest: None declared.

\section{"Quick Response Code" link for full text articles}

The journal issue has a unique new feature for reaching to the journal's website without typing a single letter. Each article on its first page has a "Quick Response Code". Using any mobile or other hand-held device with camera and GPRS/other internet source, one can reach to the full text of that particular article on the journal's website. Start a QR-code reading software (see list of free applications from http://tinyurl.com/ yzlh2tc) and point the camera to the QR-code printed in the journal. It will automatically take you to the HTML full text of that article. One can also use a desktop or laptop with web camera for similar functionality. See http://tinyurl.com/2bw7fn3 or http://tinyurl.com/3ysr3me for the free applications. 